\title{
Application Strategies of Photoelectric Detection in Mechanical Design and Manufacture
}

\author{
Hui Zhang ${ }^{1, a}$ \\ ${ }^{1}$ School of Electro-Optical Engineering, Changchun University of Science and Technology, \\ Changchun, 130022, China \\ ${ }^{\mathrm{a}}$ email
}

Keywords: Photoelectric detection, Mechanical design and manufacture, Application strategies

\begin{abstract}
Photoelectric detection technology has the characteristics of simple, fast and high security, which can greatly improve the work efficiency. Therefore, the technology is more and more favored by the manufacturing enterprises. The applications of the photoelectric detection technology in the fields of design and manufacture of printing machine packing machine and washing machine are explored in the paper. It also gives the key points of application of photoelectric detection in mechanical design and manufacture, including photocell selection, interference prevention, serviceable parts to provide some references for the relative researchers.
\end{abstract}

\section{Introduction}

In recent years, the rapid development of electronic technology, also led to the development of a variety of weak physical measurement, such as weak light, weak current, micro wave, micro vibration and so on. Detection method for most of its electricity conversion through the sensor, the measurement object is converted into electricity, method of measuring points are many, such as coherent measurement, computer processing method, discrete signal statistical average method. But the detection sensitivity and accuracy are not very ideal, therefore, scientists are devoting time to further study on the photoacoustic and photo thermal detection technology, resulting in a new development, when the need for material, light, heat, sound, magnetic force analysis and research, as long as through the detection of acoustic and thermal effect can be achieved. This technique is so useful that even subsurface nondestructive testing and imaging can be detected. The traditional light source photo thermal and photoacoustic effect is light, microwave, electromagnetic wave, has now expanded to synchrotron radiation, ion beam, electron beam, the detector is a microphone, now detector photosensitive sensor, pressure sensor and pyroelectric detector, so as to meet the needs of high technology for the detection and analysis of different applications the occasion. Light that can be used as a photoelectric detector can be light emitted by natural light, such as incandescent, fluorescent or halogen lamps. With the development of optoelectronic technology, a special modulated light is commonly used. It is the light emitted by the reflected diode under the action of voltage. Because the semiconductor has no thermal inertia, it can emit light at the instant of energization, and can emit the same light as the current waveform, which greatly improves its anti-interference ability. The light tube core, will produce photo generated carriers in the reverse voltage, the conductive carriers generate photocurrent, which varies with the incident light intensity at both ends will produce light load resistance voltage with the change of light intensity, by detecting and amplifying processing to drive control system.

\section{Application of Photoelectric Detection in Mechanical Design and Manufacture}

Application of Photoelectric Detection in Printing Machine. Application of photoelectric detection technology in printing machine is the inevitable trend of the development of modern science and technology, the printing machine can only incorporate the advanced computer information technology to make the operation more simple, faster printing speed, higher working efficiency. Through practical analysis, from the current design level, photoelectric detection 
technology is used in the printing press, the main use of its transmission photoelectric tube for position and paper detection. But to put the application of photoelectric detection technology to some small printing machine is not easy, first in the price is more expensive, and some small printing machine the enterprise scale is not too large, is difficult to bear for such expenses. From the current point of view, there are also some domestic enterprises to carry out independent research and development for printing machine technology, invented some cheap professional control devices, but these devices have a lot of shortcomings, poor operability, poor stability, safety is the most important performance remains to be studied. The photoelectric tube is groove shaped light evil general, control disc is used to control a luminous tube between the tube and the receiving tube on and off, the control disc is fixed on the rotary shaft, which can be used to detect the relative position of the rotary shaft, and a corresponding control signal according to the process requirements. The signal is sent through the light emitting sheet reaches the receiving tube, the thickness of the paper or transparency after the change, the received signal strength tube output have also changed, so with a piece of paper after commissioning, when there are two pieces of paper or paper through a luminous tube and a receiving tube, receiving tube reduced output signal strength thus, a double or more than one control signal. When the paper reaches the photoelectric tube, the reflection signal on the paper is returned to the receiving tube of the photoelectric tube, thereby producing a paper to output signal in the peripheral circuit of the receiving pipe.

Application of Photoelectric Detection in Packing Machine. The photoelectric detection technology application in the manufacturing of packaging machinery, is mainly used to calculate the color, quality testing, surface control, container positioning, is characterized not by contact can be detected, can put into static mechanical dynamic detection of photoelectric detection, which simplifies the mechanical structure, called automatic control eyes. But compared with human eyes, this structure cannot be done by human eyes in any function. For example, in response to the speed, even if the test is moving high speed, but still cannot escape the observation of this system detection function, we can see that the response speed of this detection function is fast. Therefore, the photoelectric detection technology is widely used in the manufacture of a variety of packaging machinery, such as photoelectric detection technology used in toothpaste filling machine, toothpaste tube end tail is flat, and the trademark should be always in a plane, and this is the time to use the reflection type photoelectric control device, when the toothpaste after the completion of filling, will immediately sent to the station, the cam will put toothpaste with the base from the bracket directly up through the lever, then stepping motor will drive slowly rotating it, when the toothpaste tube represents the mark and position of photoelectric trademark accurately on the receiving head together, the colored markers not reflected light, then by the light is to receive light signal is converted into electrical signals, after amplification shaping treatment of stepping motor is controlled to immediately stop running, then The flat end of the toothpaste will be in the same plane as the trademark. The photoelectric detection technology to engineering enterprise information resources integration, and maximize the utilization, through the application of high-tech electronic computer, saving staff time, improve the efficiency of enterprises.

Application of Photoelectric Detection in Washing Machine. Fuzzy control washing machine with ordinary washing machine as the foundation, the interface circuit of $\mathrm{A} / \mathrm{D}$ converter and the corresponding increase in the hardware system of the washing machine, also increased the number of sensors, the hardware system can detect some parameters of laundry, such as clothing, fabric texture, weight, washing dose, but also detected water washing machine temperature and water level information, this information can be used as the basis of fuzzy reasoning and calculation, infer the washing method of washing clothes with what is the best method. The process of washing, the photoelectric sensor can change on the turbidity of the washing liquid and water were detected, and then determine the washing time and washing machine how long and what the end of the washing, to accurately control computer. Clothing weight and clothing material is the most important two inputs of fuzzy control for washing machine. It has a direct impact on the length of washing time, water level and the amount of laundry detergent. For detecting laundry weight, calculate the weight of clothing using the method of speed measurement by power and the number of turns, the detection is based on 
the distribution of different amount of cloth produced impedance different, the motor stops off time after the cylinder, in order to adapt to different types of motor, we directly measure and drum connected the belt wheel speed and the six photoelectric sensors in water under the condition of uniform in the washing machine, the pulley rotates, the sensor emits photoelectric signal by photoelectric receiving device to collect signals measured every two adjacent sensor receives the pulse number and time interval of photoelectric signal, by the corresponding software to calculate the motor after a power speed and the number of turns, and then calculate the weight of clothing. Detection of clothing materials by detecting multi-point water level measurement, and through the power cut after measuring the rotation speed and the number of turns, and then calculate the clothing material method.

\section{Key Points of Application of Photoelectric Detection in Mechanical Design and Manufacture}

Photocell Selection. The gas filled photovoltaic is composed of a photocathode and an anode enclosed in the gas filled tube. It is different from the vacuum photoelectric tube, when the photoelectron moves to the anode under the action of the electric field and the gas atoms collide in the tube and ionization phenomenon occurs. The electrons and photoelectrons produced by ionization are both received by the anode, while the positive ions are reversely moved by the cathode. Therefore, the photocurrent in the anode circuit is several times more than that of the vacuum photocell. The electrode structure of the gas filled photocell is also different from that of the vacuum photoelectric tube. The common electrode structures are central cathode type, half cylinder cathode type and flat cathode type. The biggest drawback of the gas filled photocell is that the sensitivity declines rapidly during the working process because the positive ion bombardment the cathode and destroy the structure of the emitting layer. The gas filled tube can be divided into pure gas type and mixed gas type according to the inflation in the tube. Through the above analysis we can see that the reflective or missive photoelectric tube can be position detection, and thus to the position detection, which can be used under normal circumstances, the transmission type photoelectric tube sensitivity is higher, it can make the light path is shorter, but through the photoelectric tube to be installed in on the two position, and the need for, and bring convenience to the installation and debugging. Especially the printing machine parts more, use the reflective light curse under normal circumstances, only consider the use of trans missive light in the light reflected not disaster disaster situations, such as photoelectric detection device of double sheet printing machine, one should consider the basic sensitivity of photoelectric photoelectric tube is also used. The criterion, the photoelectric tube is not more sensitive and better, because if too sensitive may produce noise signal; otherwise too late.

Interference Prevention. Interference is an important problem to be considered when designing a circuit, if the circuit in the design of the interference into consideration, it will greatly reduce the cost of development. We must first consider the environmental interference, the photoelectric tube is on its receiver optical signal and work. If there is stronger than the surrounding light source may cause photoelectric tube disoperation, so where possible should the photoelectric tube is sealed. The photoelectric tube itself also has the noise signal, sometimes despite the luminous tube light receiving tube is signal output, thus to avoid the disoperation will be set in selecting the appropriate level, in addition to the above two kinds of interference mentioned, there are parts like switch, relay to attract or disconnect when there may be noise signal, which must also be considered in the design of the. According to the electromagnetic principle, there is no electric field inside the closed hollow conductor in the electrostatic field, and the equipotential of each point in the inner conductor. With this principle, using metal such as copper or aluminum conductive material for making metal container sealed, and the ground connection, the need to protect the value of the circuit, the external electric field interference does not affect the internal circuit, in turn, the internal electric field generated by the circuit will not affect the external circuit. This method is called electrostatic shielding. For example, sensor measuring circuit, a gap is kept between the conductor inserted in a power transformer and the two side, and it is grounded, can prevent the electrostatic coupling between two windings, this method belongs to the electrostatic shielding. 
Serviceable Parts. The photoelectric observation instrument itself with high precision and maintenance complexity, professional and technical personnel with high level of equipment maintenance requirements, strict environmental repair, maintenance and repair of special means of process, the above requirements caused by photoelectric instrument repair mode guarantee limited to equipment failure back to repair a factory installed to use the lack of fault, instrument repair method and the platform in the field, led directly to the repair cannot be timely and effective photoelectric observation equipment failure, seriously affect the normal use of the user. At present, the maintenance test of photoelectric observation instrument is mainly composed of two parts, one is the optical performance testing system, the other is the electrical performance testing system. Optical detection performance mainly by artificial methods, such as measuring the visibility needs depending on the degree of tube test, measurement of the magnification to magnification of optical instruments, detection performance of this method need to transfer the transfer function of instrument used requires maintenance personnel through optical instrument maintenance and inspection training, the measurement error will be It differs from man to man. The type and variety of electrical performance testing system are various, but mainly for the development of single type instrument, and the bus technology is usually used. This situation requires the use of units for each instrument equipped with maintenance testing system, increasing the use of pressure units. The life of the photoelectric switch times as indicators, from the use of perspective the life span can reach 1 times more than support, so there is no life problem basically, but some of the photoelectric tube due to quality problems or man-made damage of photoelectric tube will affect the normal work, and this is the time to consider it can replace the problem, considering the maintainability of the product is the most basic principle of engineering product design. The degree of professional training of users in maintenance inspection system has some limitations. The detection system should be able to provide maintenance assistance decisions according to the test results and expert database, and effectively help the personnel to complete the performance debugging of the optical system.

\section{Conclusion}

Application of photoelectric detection technology is very broad, but the design team, not only to the basic knowledge of mechanical structure of the master, to the working principle of photoelectric devices have a certain understanding, so as to make the photoelectric technology play the role of itself. In addition, the photoelectric detection technology of the device is composed of machine and electricity, when in use, if there is a problem to solve in the right way, not the machine problems as the electrical problems for processing, so the technical staff must fully grasp the principle of mechanical and electrical technology, so as to solve the problems effectively.

\section{References}

[1] Bourgeois E, Londero E, Buczak K, et al. Enhanced photoelectric detection of NV magnetic resonances in diamond under dual-beam excitation[J]. Physical Review B, 2017, 95(4): 041402.

[2] Liu D, Hu Z, Hu W, et al. Two-step method for preparing all-inorganic CsPbBr 3 perovskite film and its photoelectric detection application[J]. Materials Letters, 2017, 186: 243-246.

[3] Li H, Gao J, Wang Z. Object location fire precision test technology by using intersecting photoelectric detection target[J]. Optik-International Journal for Light and Electron Optics, 2014, 125(3): 1325-1329.

[4] Ning Y, Rongbiao Z, Peifeng X, et al. Latex immunoagglutination photoelectric detection method based on microfluidic chip[J]. Chinese Journal of Scientific Instrument, 2013, 6: 027. 